\title{
The Italian Summer Students Program at Fermi National Accelerator Laboratory and other US Laboratories.
}

\author{
E. Barzi \\ Fermi National Accelerator Laboratory \\ PO Box 500, Batavia IL, 60510-5011, USA \\ E-mail: barzi@fnal.gov \\ G. Bellettini \\ Istituto Nazionale di Fisica Nucleare - Sezione di Pisa, \\ Largo B. Pontecorvo, 3, 56127 Pisa, Italy \\ Fermi National Accelerator Laboratory \\ E-mail:giorgiob@fnal.gov
}

\section{S. Donati ${ }^{1}$}

University of Pisa - Department of Physics and Istituto Nazionale di Fisica Nucleare - Sezione di Pisa

Largo B. Pontecorvo, 3, 56127 Pisa, Italy

E-mail: simone.donati@pi.infn.it

\section{Luongo}

University of Pisa - Department of Chemistry and Industrial Chemistry

Via Giuseppe Moruzzi, 13, 56124 Pisa, Italy

E-mail: carmela.1uongo@unipi.it

Since 1984 INFN and University of Pisa scientists performing experiments at Fermilab have been running a two-month summer training program for Italian students at the lab [1]. In 1984 the program involved only a few physics students from the University of Pisa, but it was later extended to other INFN groups and to engineering students. Since 2004 the program has been supported in part by the US Department of Energy (DOE) in the frame of an exchange agreement with INFN and has been run by the Cultural Association of Italians at Fermilab (CAIF, [2]). In 2007 the Sant'Anna School of Advanced Studies (Pisa) established an agreement with Fermilab to share the cost of four engineering students each year. In the 36 years of its history, the program has hosted at Fermilab approximately 550 Italian students from more than 20 Italian universities and from some non-Italian universities. In addition, in the years 2010-2020, with the support of the Italian National Institute of Astrophyics (INAF), the Italian Space Agency (ASI), and CAIF, 30 students were hosted in other US laboratories and universities. The Fermilab training programs spanned from data analysis to design and construction of particle detectors and accelerator components, R/D on superconductive elements, theory of accelerators, and analysis of astrophysical data. At the other US laboratories the offered training was on Space Science. In 2015 the University of Pisa endorsed the program as one of its own Summer Schools [3]. The interns are enrolled as Pisa students for the duration of the internship. They are required to write summary reports published in the Fermilab and University of Pisa web pages. Upon positive evaluation by a University board, students are acknowledged 6 ECTS credits. The entire program is expected to expand further under CAIF management. An agreement has been signed between ASI and CAIF, for ASI to support yearly three two-months fellowships in US space science.

40th International Conference on High Energy Physics - ICHEP2020

28 July-6 August 2020, Prague, Czech Republic (virtual meeting)

$1 \quad$ Speaker

(C) Copyright owned by the author(s) under the terms of the Creative Commons

Attribution-NonCommercial-NoDerivatives 4.0 International License (CC BY-NC-ND 4.0). 
The program is also part of the Outreach of the European Projects MUSE (H2020-MSCA-RISE-2015, GA 690835), NEWS (H2020-MSCA-RISE-2016, GA 734303), INTENSE (H2020-MSCA-RISE-2018, GA 822185) and INTENSE (H2020-MSCA-ITN-2019, GA 858199).

In the following we inform on student recruiting, training programs, and final evaluation.
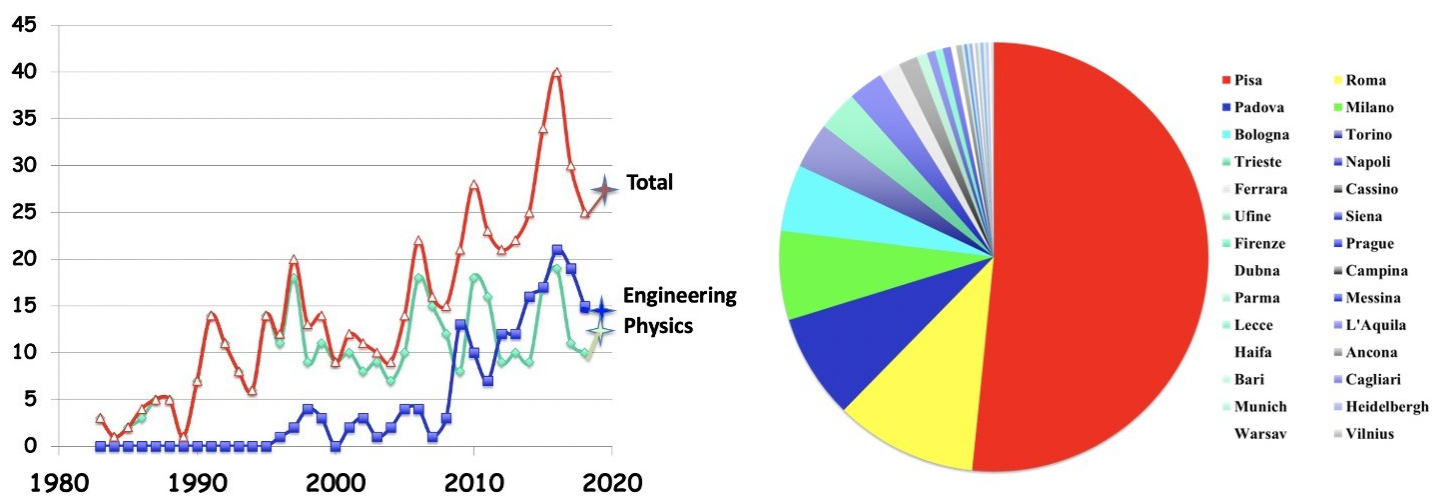

Figure 1 (left) Distribution of the number of students selected in the years 1984-2019 (the Program of the year 2020 was cancelled): the blue curve represents the distribution of the engineering students, the green curve represents the distribution of the physics students and the red curve represents the total distribution. One notes an increased rate of engineering students in recent years, who are now as many as the physics students; (right) distribution of the number of students selected from Italian and some European universities.

\section{Recruitment}

In January we announce the available grants with posters and flyers, on the web and on most Italian Universities, whereby some basic information is given to the applicants. The youngest undergraduates are excluded by requesting students of the "Laurea Magistrale" courses from Italian universities and of the Masters degree from European universities. Computer science skills and good knowledge of English are requested. In early spring CAIF members inquire about the available training programs at the lab, to be matched to the skills and interests of the trainees. One must find out which groups are interested in offering a training program to our students, and get a clear description of the offered program. It is essential to find a match between the profiles of the candidates and the interest of the groups. Only students matching an available training programs are ultimately selected. Pre-selected candidates are informed that they will have to discuss their case in person or by Skype with representatives of CAIF and of the sponsors. Thorough interviews are performed. The selected students are made known to supervisors as potential trainees in the months of April/May. The supervisors make their best choice and work offers are sent to the students. Finally, those students for whom a good fit and work program are found within a Fermilab team will enter the US with a J1 Visa for training in August and September. Free housing and shared rental cars are provided besides a weekly salary. Fermilab does not cover the round trip journey to the US and the health insurance. However, the salary is fully adequate to allow the students to cover the travel cost as well as all costs encountered in two months of stay. CAIF negotiates agreements with outside labs for training students sponsored by the Italian Space Science Agency (ASI, http://www.asi.it). As for the Fermilab students, early in the year the available ASI fellowships (currently 3/year) and the support offered by CAIF are announced, and in late spring the winners are selected. Their training period is from the end of July to the end of September. Figure 1 (left) shows the number of students selected each year from 1984 to 2019 (the Program of the year 2020 was cancelled). Figure 1 (right) shows the students' universities of origin. About $75 \%$ of the students come from the universities of Pisa, Rome, Milan and Padova. 


\section{Logistics}

The Fermilab Personnel Office receives the list of the selected students and e-mails them the job offers needed to get the J1 Visa required to be employed as Fermilab interns. Once the offer is accepted the students are responsible for getting their Visa. In addition, some paperwork is requested from the students by the Fermilab Visa Office. This includes accessing from Italy the "FermiWorks" website to complete an Onboarding process and filling a New Hire document. The rules for accessing the FERMI computing domain can also be followed from a distance, in order to gain access to the domain immediately upon arrival at the lab. At the student arrival CAIF members are around making sure that students housing proceeds smoothly in the lab dorm or in nearby hotels. On the first and the second day at the lab the students convene for an International Orientation session, where they are istructed on basic lab and US rules. A twin concluding session must be followed shortly before departure at the end of the training period. Although this bureaucracy may worry the students at the beginning, experience shows that they are able to learn quickly to fulfil all the requests, and that in a few days they are able to work efficiently in their groups, who are responsible for assigning to them adequate office space and computing power.
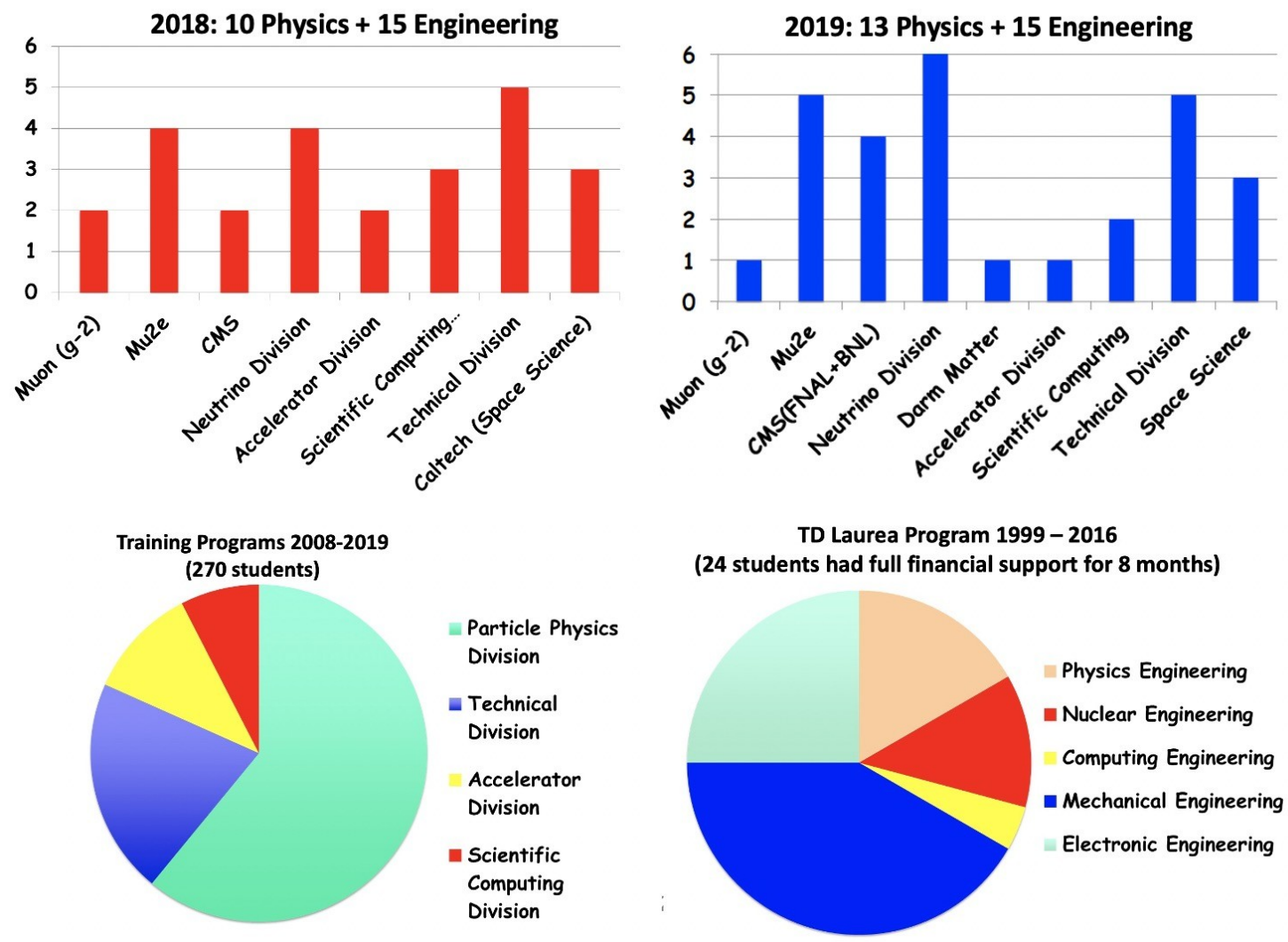

Figure 2: (Top) Students’ assignments among experimental groups in the year 2018 and 2019; (Bottom left ) Students' assignments among Fermilab Divisions in the years 2008-2019; (Bottom right) Master students in Engineering accepted in the Technical Division Laurea Program in the years 1999-2016.

\section{Work Programs}

The training programs span a very wide range of science and technology. The students are integrated in their research groups and are encouraged to interact with as many colleagues as possible. The supervisors meet with their students on an individual basis, with meetings held at least once per week, to allow the group to profit of the productivity of the students. The students also participate in collaboration meetings, 
where they present their results in a wide professional environment. For physicists, students address analysis of experimental data in particle physics and astrophysics, simulation and setting-up of particle detectors, and particle accelerator theory. For engineers, they include fast digital electronics, design of detectors and accelerator components, superconducting materials and magnets, high precision mechanics, and advanced computing as well as civil engineering projects. Students make extensive use of advanced computation means and programming languages, as $\mathrm{C}, \mathrm{C}++$, and Java, and apply advanced CAD and other technical tools for mechanics and electronics design (MatLab, OrCAD, Ansys, etc.). Physicists develop knowledge in statistical data analysis (Root). At Fermilab work is performed within projects, programs and experiments like Mu2e, Muon (g-2), NoVA, MicroBoone, LAriAT, Icarus, SBND, LBNF, DUNE, CDF, CMS, and General Accelerator R\&D. Figure 2 (Bottom left) reports the distribution of the students' assignments among the Fermilab Divisions for the programs of the years 2010 to 2019. Figure 2 (Top) shows the students' assignments to the Fermilab groups in 2018 and 2019. All students are requested to give midterm and final oral presentations and to write a technical report at the end of their stay. These documents are saved in the Fermilab Education Office web archive [4].

\section{Laurea Students}

Based on the strong interest that a number of former Italian summer students showed to come back to the lab and continue their research an official thesis program for a Laurea Magistrale was started by the lab. Some students were invited back to the lab and employed again for a period of about 8 months, as needed for performing an adequate research program for their Laurea Magistrale. Although the funds made available have been rather limited and the program was confined to the Technical Division, 24 Italian engineering students, out of which 9 had been previously summer students, were supported by the lab for their Laurea program in the years 1999-2016 (Figure 2, Bottom Right). A number of them are now permanent employees. This shows how the laboratory had a significant return from this program. In addition to the Fermilab Laurea Magistrale students, a total of more than 200 students in Physics and Engineering worked for their Laurea Triennale, Laurea Magistrale or PhD within the INFN groups involved in the CDF, Muon (g-2), Mu2e and ICARUS experiments at Fermilab. About 50\% of them were initially summer students.

\section{Internships at US Space Science Laboratories}

Outside Fermilab, work is performed in astrophysics, space science and technology. In 2010 ASI and INAF started providing financial support to CAIF for 2-month internships in US space science laboratories, similarly to the Fermilab program. Students' selection is made by CAIF members in collaboration with INAF or ASI personnel. As of now, host institutions in US have included the NASA Goddard Space Flight Center, the Space Telescope Science Institute in Baltimore, the Harvard Smithsonian Center for Astrophyscs, the NaSA Jet Propulsion Laboratory in Pasadena, Stanford University, Columbia University in New York, the University of Arizona in Tucson, the SLAC National Accelerator Laboratory, the University of Colorado at Boulder, Purdue University, and the University of Texas at Arlington. Figure 3 shows the distribution of the 30 students among US space science laboratories and universities.

\section{The University of Pisa Summer School}

In 2015 an ad-hoc summer course was approved at the University of Pisa to provide an academic framework to the Fermilab program. Interns are enrolled as University of Pisa students for the 9-week duration of the internship. They have a regular position as local students with a proper identification and insurance coverage. Upon successful completion of the internship with an accurate final report and an oral interview, they are acknowledged 6 ECTS in their Diploma Supplement (https://www.unipi.it/summerschool).

\section{Growing Involvement at European Level}


The Fermilab internship program has become part of the outreach activities of European Projects, MUSE "Muon Campus in US and Europe contribution" (H2020-MSCA-RISE-2015, GA 690835, muse.lnf.infn.it), and NEWS "NEw WindowS on the Universe and technological advancements from trilateral EU-US-Japan collaboration” (H2020-MSCA-RISE-2016, GA 734303, risenews.df.unipi.it), INTENSE "INTENSE: particle physics experiments at the high intensity frontier, from new physics to spin-offs. A cooperative Europe - United States - Japan effort” (H2020-MSCA-RISE-2p018, GA 822185, riseintense.df.unipi.it), and INTENSE "INTENSE: particle physics experiments at the intensity frontier. A cooperative Europe - United States effort” (H2020-MSCA-ITN-2019, GA 858199, itnintense.df.unipi.it). INFN and the University of Pisa coordinate these European Projects, which count a large number of universities, research institutes and private companies in Europe, United States and Asia. The EU provides financial support for the mobility of European researchers to the US and of Japan participants. Involved researchers have organized seminars and short courses on the topics of their research activities for the Fermilab intern.

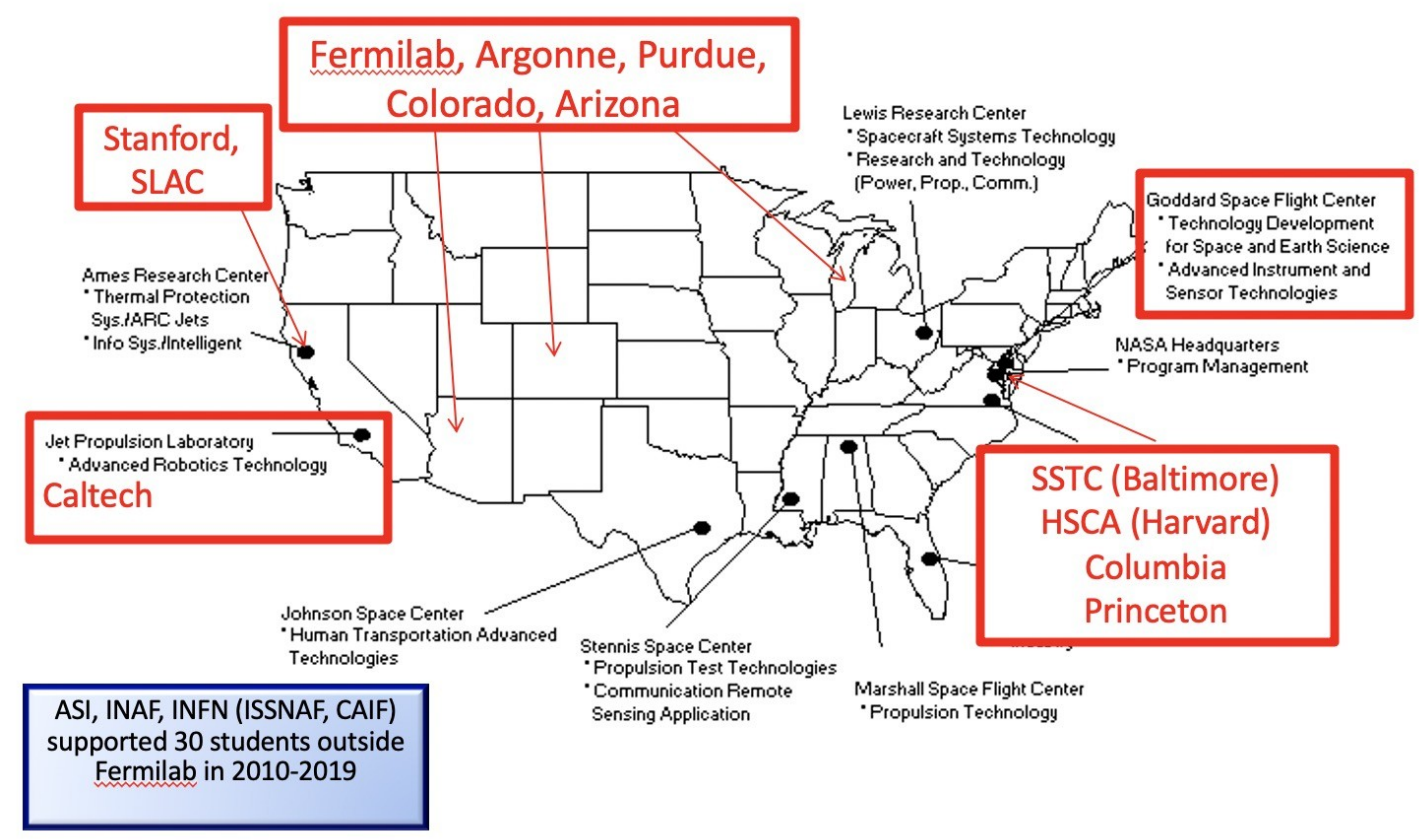

Figure 3: Space Science laboratories selected by the students for the INAF and ASI fellowships.

\section{Conclusions}

A voluntary, self-managed education program started long ago by a few Italian physicists at INFN and the University of Pisa engaged in particle physics experiments at Fermilab in the US has turned into a solid, multi-disciplinary program bringing dozens of bright young undergraduate and graduate physicists and engineers to learn and contribute in high-tech research in the US every year. Over the thirty-five years of its life (1984-2020), more than 550 students have taken part in the program. Stimulated by the enthusiastic reports of their students, Italian universities now convey a strong feeling of appreciation for this program.

\section{Acknowledments}

Many colleagues at Fermilab and elsewhere have contributed to the success of this program by supervising the students and guiding them to take full advantage of the lab's capabilities. Their role has been and 
will be vital. The program would not have been possible without the strong support of the Lab Chief Operating Officer Timothy Meyer and the highly professional and friendly help of the Visa and the Education Offices. We are grateful to INFN and ASI for their support to CAIS which provides the means for a vital and active Associatgion.

This work was supported by the EU Horizon 2020 Research and Innovation Programme under the Marie Sklodowska-Curie Grant Agreement No. 690835, 734303, 822185, 858199.

\section{References}

[1] E. Barzi, G. Bellettini, S. Donati, “The Italian Summer Students Program at the Fermi National Acelerator Laboratory”, Proceedings of European Physical Society Conference on High Energy Physics, Venice, Italy, 5-12 July 2017; E. Barzi, G. Bellettini, S. Donati, C. Luongo, "The Italian Summer Students Program at the Fermi National Accelerator Laboratory and other US Laboratories”, Proceedings of the XXXIX International Conference on High Energy Physics (ICHEP 2018), Seoul, Korea, July 4-11, 2018; E. Barzi, G. Bellettini, S. Donati, C. Luongo, "The Italian Summer Students Program at the Fermi National Accelerator Laboratory and other US Laboratories”, Proceedings of the 2019 Meeting of the Division of Particle and Fields of the American Physical Society, Northeastern University, Boston MA (US), July 29 - August 2, 2019.

[2] http://caif.fnal.gov; https://www.primapagina.sif.it/article/648/the-cultural-association-of-italians-atfermilab.

[3] https://www.unipi.it/summerschool.

[4] http://eddata.fnal.gov/lasso/summerstudents/view.lasso. 
Title (or short title) 